\title{
Prompt fission neutron and $\gamma$ ray properties as a function of incident neutron energy
}

\author{
Ionel Stetcu ${ }^{\mathrm{a}}$, Patrick Talou, and Toshihiko Kawano \\ Theoretical Division, Los Alamos National Laboratory, PO Box 1663, MS B283, Los Alamos, New Mexico, USA
}

\begin{abstract}
The CGMF code provides a complete description of the properties of the prompt neutrons and $\gamma$ rays, emitted before beta decays. It is based on a Monte Carlo implementation of the Hauser-Feshbach statistical model, which provides an accurate phenomenological description of the de-excitation of the fission fragments toward stable configurations via neutron and $\gamma$-ray emissions. This approach allows a detailed description of a large number of observables, such as multiplicity probabilities and correlations between the emitted particles. In this contribution, we briefly review the approach and present selected examples of neutron and $\gamma$-ray observables for neutron incident energies from thermal to $20 \mathrm{MeV}$, and compare against available data for ${ }^{235} \mathrm{U}(\mathrm{n}, \mathrm{f})$ reaction.
\end{abstract}

\section{Introduction}

Nuclear reactors and other applications often require the knowledge of the energy released in neutron-induced fission reactions via the emission of prompt neutrons and $\gamma$ rays. In other applications, correlations between the prompt particles are of particular interest. Hence, in this contribution we describe the implementation of the incident neutron energy into the CGMF (Cascading Gamma-ray and Multiplicity for Fission) code developed at Los Alamos for simulating prompt neutron and $\gamma$-ray observables. The neutron-induced fission of ${ }^{235,238} \mathrm{U}$ and ${ }^{239} \mathrm{Pu}$, and the spontaneous fission of ${ }^{240,242} \mathrm{Pu}$ and ${ }^{252} \mathrm{Cf}$ can be currently simulated in CGMF, while other reactions, including photofission, are planned to be implemented in the near future.

In fission, the daughter fragments are produced with excitation energy around $20-30 \mathrm{MeV}$, which is then mainly released via neutrons and $\gamma$ rays. Thus, the fragments can be treated as compound nuclei, whose de-excitation toward stable configurations is modeled in the Hauser-Feshbach statistical decay model [1], that provides a detailed phenomenological description of the de-excitation process. A large number of properties of far-from-stability nuclei, from level densities to neutron transmission coefficients or electromagnetic decay properties, is necessary as input for such a sophisticated approach. However, the number of observables that can be described is also large, from average quantities (e.g., average neutron or $\gamma$-ray multiplicity per fission process) to distributions or correlations between the prompt particles. Similar codes that treat the de-excitation fission fragments via emission of neutrons and $\gamma$ rays are available in the literature [2-4].

\section{Theoretical model}

A first step in the simulation is generating a collection of fission fragments (FFs) with defined excitation energy, angular momenta, parity and kinetic energy. Thus, CGMF requires a priori knowledge of the yields in mass, charge and total kinetic energy (TKE), $Y(A, Z, \mathrm{TKE})$, as a function of incident neutron energy. $Y(A, Z, \mathrm{TKE})$ is not modeled in CGMF, but rather taken either directly from experiment or from systematics. In our implementation, we make the assumption that the yields in mass, charge and TKE can be expressed as

$Y\left(A, Z, \mathrm{TKE} ; E_{n}\right)=Y\left(A ; E_{n}\right) Y\left(Z \mid A ; E_{n}\right) Y\left(\mathrm{TKE} \mid A ; E_{n}\right)$,

where $E_{n}$ is the energy of the incident neutron. More detailed discussions can be found in Ref. [5], and in another paper currently in preparation.

Inspired by the neck rupture model [6], we assume that the mass yields are given as a superposition of three fission modes (two asymmetric and one symmetric), each fission mode being characterized by Gaussian with an average mass and width. The incident neutron energy dependence of the parameters characterizing each mode is taken as in Ref. [4]. Since the charge distributions are not changed by the neutron emissions, the charge yields $Y\left(Z \mid A ; E_{n}\right)$ are constructed from the post-neutron emission systematics developed by Wahl [7].

The yields $Y\left(\mathrm{TKE} \mid A ; E_{n}\right)$ play an important role in the correct description of neutron observables, as they determine to a large extent the number of prompt neutrons emitted in fission. In the current implementation, we assume that for each fragment mass pair, the TKE distribution is a Gaussian characterized by $\overline{\operatorname{TKE}}\left(A ; E_{n}\right)$ and width $\sigma_{\mathrm{TKE}}\left(A ; E_{n}\right)$. The high-TKE events, which are not allowed by the Q-value of the reaction, are discarded.

Not much data on $\overline{\operatorname{TKE}}\left(A ; E_{n}\right)$ and $\sigma_{\mathrm{TKE}}\left(A ; E_{n}\right)$, especially as a function of incident neutron energy,

\footnotetext{
a e-mail: stetcu@lanl.gov
}

(C) The Authors, published by EDP Sciences. This is an Open Access article distributed under the terms of the Creative Commons Attribution License 4.0 (http://creativecommons.org/licenses/by/4.0/). 


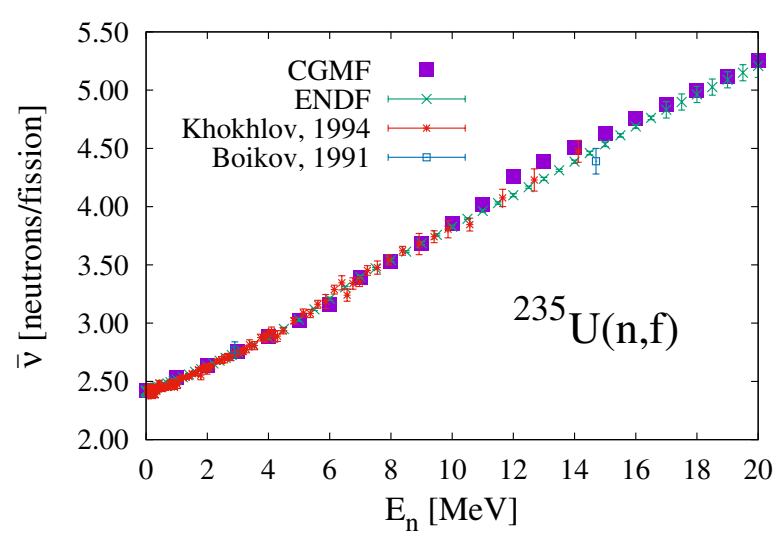

Figure 1. Incident neutron energy dependence of the average number of prompt neutrons per fission.

are available at the moment for the reactions of interest. However, it was noted before (see Fig. 1(a) in Ref. [5]), as a first-order approximation, that most of the energy dependence of $\operatorname{TKE}\left(A ; E_{n}\right)$ can be encoded in the energy dependence of $\overline{\mathrm{TKE}}$ for the reaction, which is mathematically equivalent to a factorization $\overline{\operatorname{TKE}}\left(A, E_{n}\right)=C\left(E_{n}\right) \cdot \operatorname{TKE}\left(A, E_{\text {ref }}\right)$, where $E_{\text {ref }}$ is a neutron-incident energy for which experimental data on $\overline{\operatorname{TKE}}(A)$ exists. This factorization seems to hold for a large number of isotopes, where data at different neutron incident energies are available. TKE has been measured, most recently at Los Alamos Neutron Science Center (LANSCE), and below the threshold for second chance fission, one usually finds a linear decrease of $\overline{\mathrm{TKE}}$ with $E_{n}$ [8]. One exception is ${ }^{235} \mathrm{U}(\mathrm{n}, \mathrm{f})$, where several experiments point to a slight increase of TKE with $E_{n}$ from thermal to $1 \mathrm{MeV}$, but above that the familiar linear decrease with incident energy reemerges. In the current implementation, we assume a linear decrease of $\overline{\mathrm{TKE}}$ with energy for each fissioning system up to $20 \mathrm{MeV}$, with the intercept and slope adjusted in each case so that we reproduce to a good approximation the data on $\overline{\operatorname{TKE}}\left(E_{n}\right)$. Only in the case of the ${ }^{235} \mathrm{U}(\mathrm{n}, \mathrm{f})$ reaction, we consider a linear increase of the average TKE from thermal to $1 \mathrm{MeV}$. The proportionality constant $C\left(E_{n}\right)$ is calculated simply from the relation $\overline{\operatorname{TKE}}\left(E_{n}\right)=C\left(E_{n}\right) \sum_{A} Y\left(A ; E_{n}\right) \overline{\operatorname{TKE}}\left(A ; E_{n}\right)$, where the summation is performed over the light (or heavy) FFs.

The width of the TKE distribution was kept independent of energy, as suggested by experimental data (see Fig. 1(b) in Ref. [5]). However, in order to more realistically reproduce the neutron multiplicity distribution at low energies, all the values of $\sigma_{\mathrm{TKE}}\left(A ; E_{n}\right)$ have been reduced by 20 to $25 \%$ from the experimental values reported in the literature, depending on the reaction.

At higher incident neutron energy, one or more neutrons can be emitted before fission. In that case, the fissioning system is different from the original compound nucleus, and has a lower excitation energy. For each event, we determine the fissioning system by sampling the multi-chance fission probabilities to determine the number of neutrons emitted before fission. The multichance fission probabilities, which are not observables, are obtained from double-hump fission barrier penetrability calculations. The pre-fission neutron energies are sampled from the compound and pre-equilibrium neutron spectra.

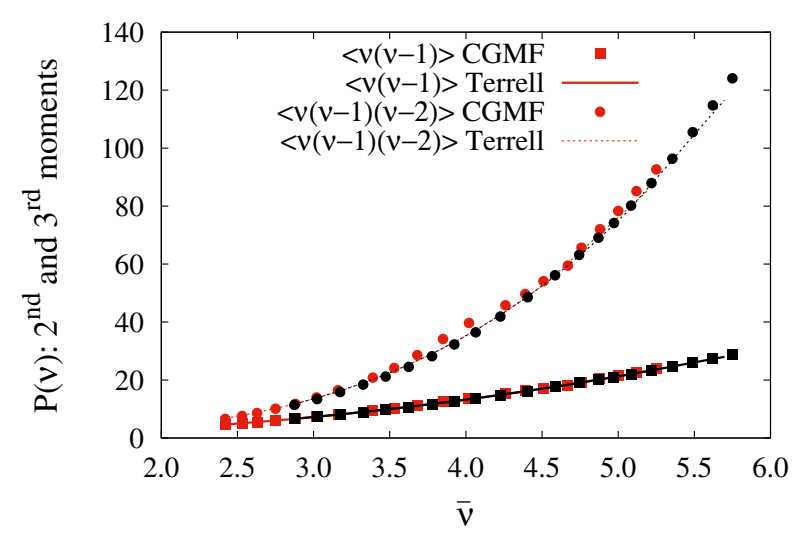

Figure 2. The second and third moment of the multiplicity probability distribution as a function of the average neutron multiplicity. The CGMF results are displayed with symbols, and Terrell's evaluations with lines, in red for ${ }^{235} \mathrm{U}(\mathrm{n}, \mathrm{f})$ and in black for ${ }^{239} \mathrm{Pu}(\mathrm{n}, \mathrm{f})$.

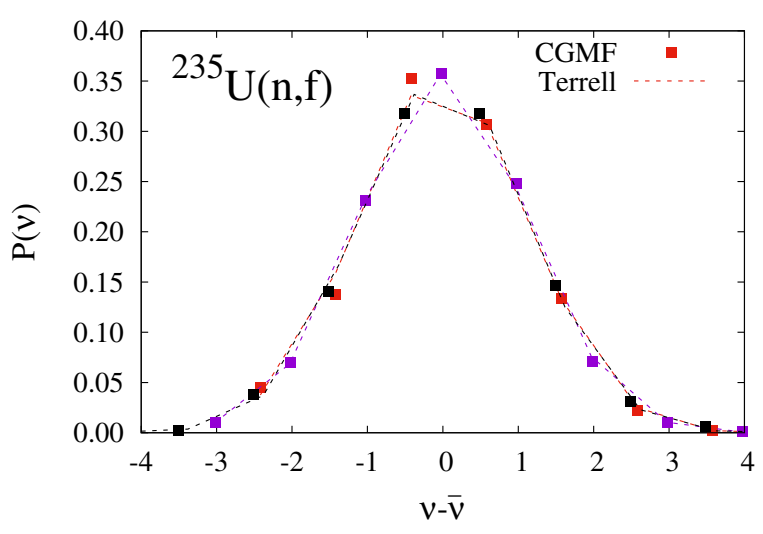

Figure 3. The multiplicity probability distribution for the ${ }^{235} \mathrm{U}(\mathrm{n}, \mathrm{f})$ reactions, at thermal (red), $5 \mathrm{MeV}$ (purple), and $14 \mathrm{MeV}$ (black) neutron incident energies. The CGMF results are shown with symbols, while the Terrell evaluations is displayed with dashed lines).

If the remaining excitation energy in the fissioning system is below the fission barrier, we reject that event, as fission is strongly suppressed. We should emphasize that the multi-chance fission probabilities and fission barriers are not observables. Hence, the sensitivity to these will have to be closely investigated. The mass, charge, and TKE yields are determined event-by-event, for each fissioning system, and are parameterized in terms of the "equivalent" incident neutron energy, which is defined as the energy of a fictitious neutron incident on a $\mathcal{A}-1$ target, which would produce the same excitation energy of the fissioning $\mathcal{A}$ system.

Using $Y\left(A, Z, \mathrm{TKE} ; E_{n}\right)$, we perform a Monte-Carlo sampling of pairs of FFs with defined TKE. From the energetics of the reaction, we determine the total excitation energy (TXE) available to both fragments. The sharing mechanism is parameterized in terms of massdependent ratios of heavy and light temperatures that are fitted to reproduce the ratio of prompt neutrons emitted from light and heavy fragments respectively $[9,10]$. However, because we have performed fits only at thermal incident neutron energy, further refinement for the energy dependence of the energy-sharing parameterization is required. 

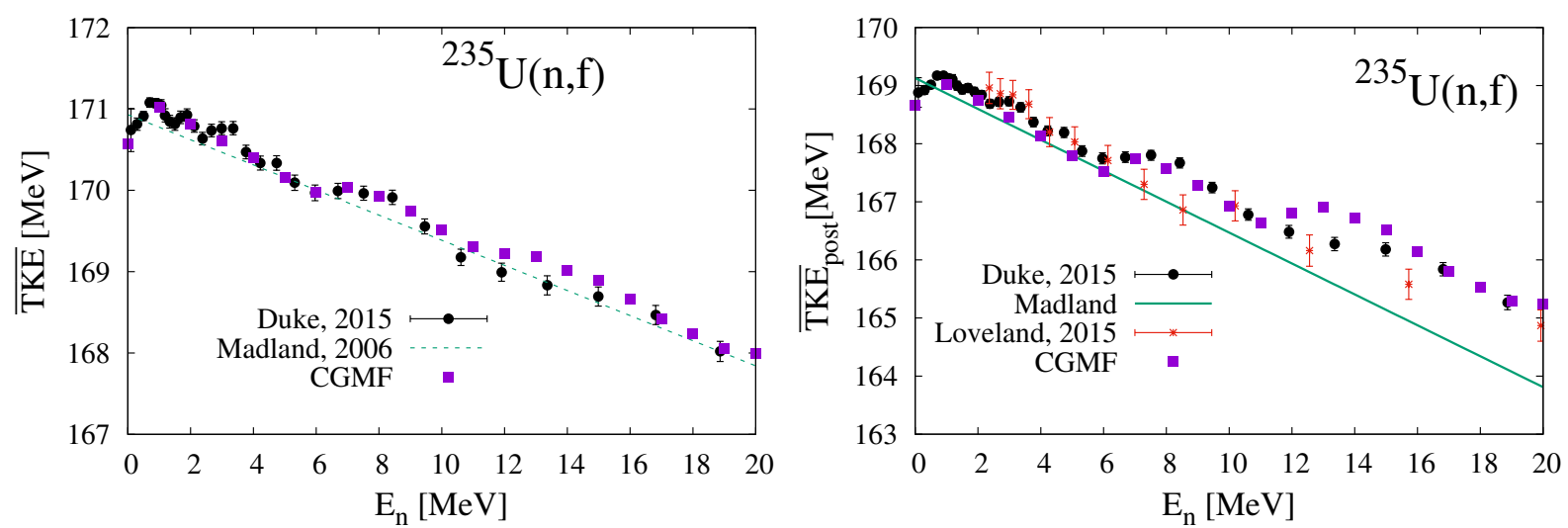

Figure 4. The average TKE as a function of neutron incident energy for the ${ }^{235} \mathrm{U}(\mathrm{n}, \mathrm{f})$ reaction. The CGMF results are compared with data from the LANSCE measurement and the Madland systematics. We show both pre-neutron (left panel) and post-neutron (right panel) emission $\overline{\mathrm{TKE}}$.

In competition with neutron emissions, the release of energy from FFs via $\gamma$ rays is modeled in the same Hauser-Feshbach framework, by constructing the $\gamma$ transmission coefficients from the strength function via the Kopecky-Uhl formalism [11]. Only E1, M1 and E2 multipolarities are taken into account, while for transitions between discrete levels, we use the experimental branching ratios available in the RIPL3 database [12]. The results depend on the spin of the FFs, which in turn is determined in our simulations by a global parameter $\alpha$ (see our previous publications $[10,13]$ ). This parameter is adjusted to fine tune the average neutron multiplicity to ENDF values below $5.5 \mathrm{MeV}$. Other ingredients that play a major role in our calculations, are also discussed in previous publications $[10,13]$.

\section{Selected results}

The space limitation do not allow us to present a large number of results. Hence, in Figs. $1-5$ we show only a small selection of observables.

In Fig. 1 we present the results of our calculations for the average neutrons emitted per fission event, $\bar{v}$, as a function of incident neutron energy. As noted in Sect. 2, we have adjusted both the TKE and FF spin dependences of $E_{n}$ to reproduce the average number of prompt neutrons per fission below the threshold for the second-chance fission. Above this threshold, without further adjustments, our results are in reasonable agreement with the evaluated data, in many cases within the expected uncertainties. The only energy region in which our simulations are in discrepancy with the evaluations is 12 to $14 \mathrm{MeV}$. While it is reasonable to assume that the evaluated errors are underestimated, the almost 5\% discrepancy is large enough to suggest that further improvements of our model are necessary. It is conceivable that, because the discrepancy appear around the threshold for third-chance fission, at least part of the problem resides in the multi-chance fission probabilities, which are not observables. However, the sensitivity of neutron observables to multichance fission probabilities is not large enough (at least in the case of ${ }^{239} \mathrm{Pu}(\mathrm{n}, \mathrm{f})$ reaction, where we have performed several studies), to explain the disagreement. Hence, other simplifications in our model, e.g., the average spin of FFs, have to be investigated more thoroughly. The second and third moments of the prompt neutron distribution as a function of average neutron multiplicity are presented in Fig. 2 in comparison with Terrell's evaluation [14] for both ${ }^{235} \mathrm{U}(\mathrm{n}, \mathrm{f})$ and ${ }^{239} \mathrm{Pu}(\mathrm{n}, \mathrm{f})$ reactions. The second moment is within $10 \%$ of Terrell's evaluation, while the third moment presents up to a $20 \%$ discrepancy. The largest discrepancies, as in the case of $\bar{v}$ are for incident energies of 12 to $14 \mathrm{MeV}$. The multiplicity probability distributions for thermal, $5 \mathrm{MeV}$, and $14 \mathrm{MeV}$ are presented in Fig. 3. While the agreement is reasonable, for some applications the accuracy is not high enough. This suggest that further investigations of the width of the TKE distributions as a function of mass are necessary, as this quantity plays an important role for $P(v)$.

The same striking discrepancy between our results and experimental data around 12 to $14 \mathrm{MeV}$ incident-neutron energy is visible again in Fig. 4, for both pre- and postneutron emission average TKE in the case of ${ }^{235} \mathrm{U}(\mathrm{n}, \mathrm{f})$ reaction. Note that, while several TKE (intercept and slope of the TKE dependence in the fissioning system) adjustments are made around the multi-chance fission, the post-neutron emission TKE in the right hand panel is a direct results of the simulations, without further adjustments. Finally, in order to obtain the correct $\bar{v}$ at reasonable values of FF spins, we have increased at all incident energies the average TKE. Because for ${ }^{235} \mathrm{U}(\mathrm{n}, \mathrm{f})$ we have increased $\overline{\operatorname{TKE}}\left(E_{n}\right)$ by $0.9 \mathrm{MeV}$, in Fig. 4 our TKE values have been reduced by $0.9 \mathrm{MeV}$, otherwise a direct comparison of the trend with experimental data would be difficult. Thus, excluding this overall shift, which can be attributed to uncertainties in the absolute measurement, the overall trend is close to the experimental data.

Much less experimental data is available for $\gamma$-ray observables above thermal incident energy. In Fig. 5 we show the average total $\gamma$ energy $E_{\text {tot }}^{\gamma}$ in ratio to the same quantity for ${ }^{252} \mathrm{Cf}(\mathrm{sf})$, and compare it against data by Frehaut [15]. We should emphasize that in the same experiment $\bar{v}$ was measured as well, with rather large discrepancies from today's accepted values. Note that in our simulations $E_{\text {tot }}^{\gamma}$ decreases from thermal to $1 \mathrm{MeV}$ incident energy. This is due to an increase in TKE between thermal and $1 \mathrm{MeV}$ incident energy observed in all experiments, including the most recent at the Los Alamos Neutron Science Center [16]. In our calculations, we implement this rise in $\overline{\mathrm{TKE}}$, which in turn requires a drop in FF spins in order to reproduce $\bar{\nu}$. The slope in $E_{t o t}^{\gamma}$ is determined by interplay between the slope of $\overline{\operatorname{TKE}}\left(E_{n}\right)$ 


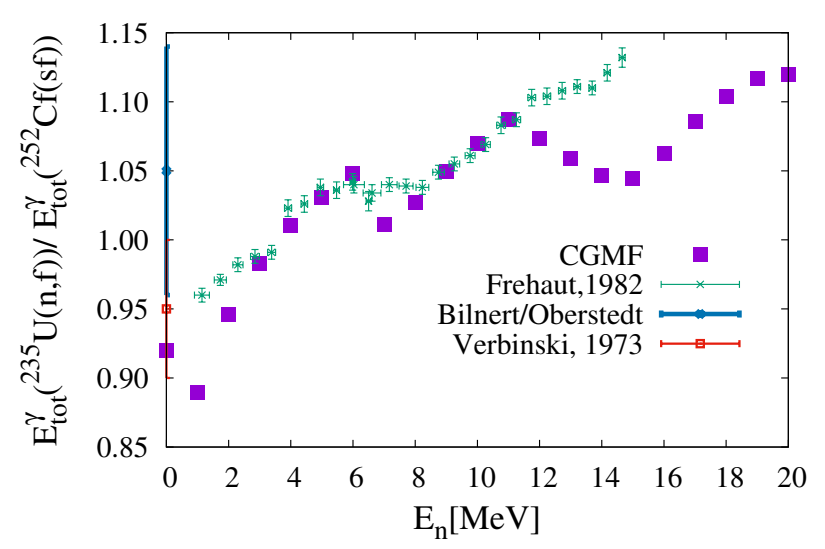

Figure 5. Incident neutron energy dependence of the average total $\gamma$-ray energy in ratio to average total prompt $\gamma$-ray energy emitted in ${ }^{252} \mathrm{Cf}$.

and FF spin. Future work will investigate whether small changes in TKE dependence on $E_{n}$ can better reproduce $E_{\text {tot }}^{\gamma}\left(E_{n}\right)$.

\section{Conclusion}

We have presented our Monte Carlo implementation of the Hauser-Feshbach statistical model to the de-excitation of FFs produced in the neutron induced fission of actinides. Due to the space limitation, we have illustrated the incident neutron energy dependence of only a few observables for the ${ }^{235} \mathrm{U}(\mathrm{n}, \mathrm{f})$ reaction. In general, we reproduce reasonably well $\bar{v}$ up to $20 \mathrm{MeV}$. Average TKE, pre- and post-neutron emission, is also consistent with available data and current models. The data on $\gamma$-ray observables point to some issues in our simulation, so that we will continue the investigations on different models and parameterizations employed in CGMF. However, future well-documented experiments can better help constrain our calculations.

This work was performed at Los Alamos National Laboratory, under the auspices of the National Nuclear Security Administration of the U.S. Department of Energy at Los Alamos National
Laboratory under contract No. DE-AC52-06NA25396. We acknowledge partial support from the Office of Defense Nuclear Nonproliferation Research \& Development (DNN R\&D), National Nuclear Security Administration, US Department of Energy.

\section{References}

[1] W. Hauser, H. Feshbach, Phys. Rev. 87, 366 (1952)

[2] K.H. Schmidt, B. Jurado, C. Amouroux, C. Schmitt, Nuclear Data Sheets 131, 107 (2016)

[3] D. Regnier, Ph.D. thesis, Université de Grenoble (2013)

[4] R. Vogt, J. Randrup, D.A. Brown, M.A. Descalle, W.E. Ormand, Phys. Rev. C 85, 024608 (2012)

[5] I. Stetcu, P. Talou, T. Kawano, EPJ Web of Conferences 122, 01012 (2016)

[6] U. Brosa, S. Grossmann, A. Müller, Physics Reports 197, 167 (1990)

[7] A.C. Wahl, Report LA-13928, Los Alamos National Laboratory (2002)

[8] D. Madland, Nuclear Physics A 772, 113 (2006)

[9] P. Talou, B. Becker, T. Kawano, M.B. Chadwick, Y. Danon, Phys. Rev. C 83, 064612 (2011)

[10] B. Becker, P. Talou, T. Kawano, Y. Danon, I. Stetcu, Phys. Rev. C 87, 014617 (2013)

[11] J. Kopecky, M. Uhl, Phys. Rev. C 41, 1941 (1990)

[12] R. Capote, M. Herman, P. Obložinský, P. Young, S. Goriely, T. Belgya, A. Ignatyuk, A. Koning, S. Hilaire, V. Plujko et al., Nucl. Data Sheets 110, 3107 (2009), special Issue on Nuclear Reaction Data

[13] I. Stetcu, P. Talou, T. Kawano, M. Jandel, Phys. Rev. C 90, 024617 (2014)

[14] J. Terrell, Phys. Rev. 108, 783 (1957)

[15] J. Fréhaut, A. Bertin, R. Bois, Mesure de $\bar{v}_{p}$ pour la fission de ${ }^{232} \mathrm{Th},{ }^{235} \mathrm{U}$ et ${ }^{237} \mathrm{~Np}$ induite par des neutrons d'energie comprise entre 1 et $15 \mathrm{MeV}$, in International Conference on Nuclear Data for Science and Technology, Antwerp, Belgium (Reidel, Dordrech, Holland, 1983), p. 78

[16] D.L. Duke, Tech. Rep. LA-UR-15-28829, Los Alamos National Laboratory (2015) 